\title{
A new drug for resistant bugs
}

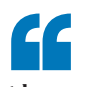

the authors

were unable

to isolate

teixobactin-

resistant

mutants

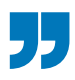

The paucity of novel antimicrobial compounds in the drug discovery pipeline is one of the main hurdles to combatting the current antibiotic resistance crisis. Now, Lewis and colleagues report the discovery of the new antibiotic teixobactin, which has a completely novel mechanism of action and does not seem to select for resistance.

Most antibiotics in clinical use were discovered by screening cultivable soil microorganisms, but this resource is limited and alternative synthetic approaches have had little success. As up to $99 \%$ of environmental bacteria are uncultured and are a potentially untapped reservoir of novel compounds, the authors

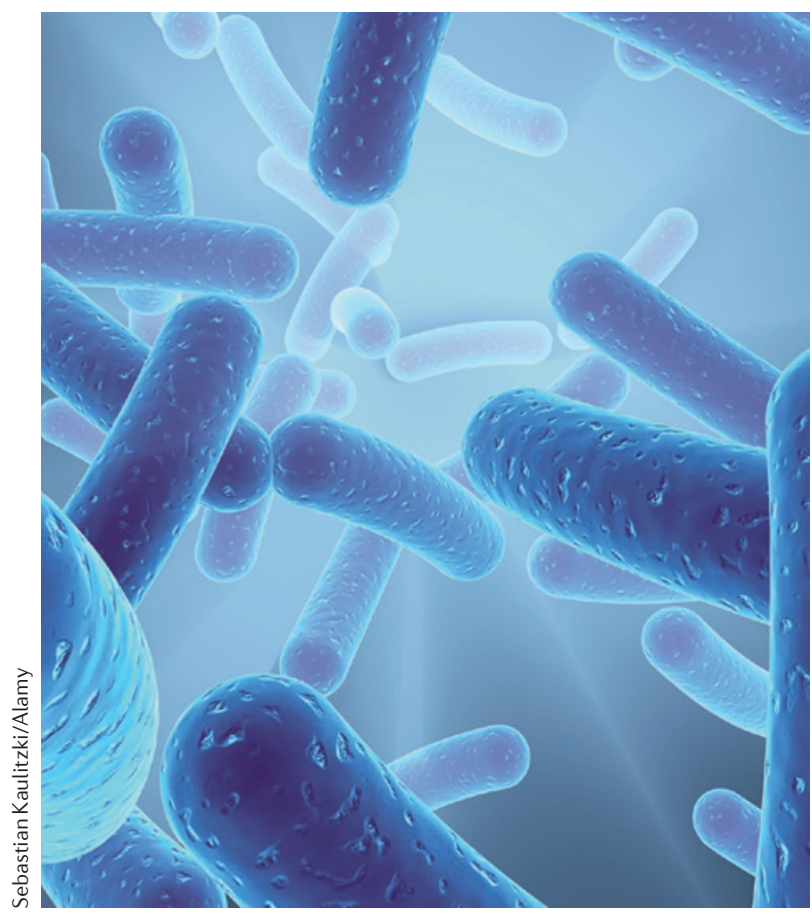

developed a high-throughput, multichannel device called the iChip to screen previously uncultured soil bacteria. This approach involves isolating an individual bacterial cell in one of 96 chambers that is surrounded by a semi-permeable membrane; bacteria can then be grown in situ as they have access to the nurients and growth factors present in their natural environment.

The authors examined the antimicrobial activity of extracts obtained from 10,000 soil bacteria and found that an extract from a new species of the Gram-negative $\beta$-proteobacteria, provisionally named Eleftheria terrae, showed promising inhibitory activity against the Gram-positive pathogen Staphylococcus aureus. Structural analysis showed that the antimicrobial compound, termed teixobactin, has a unique chemical scaffold and thus represents a completely new antibiotic class.

In addition to $S$. aureus, teixobactin is a potent inhibitor of other drug-resistant Gram-positive pathogens, including Mycobacterium tuberculosis, Clostridium difficile and Bacillus anthracis, but is ineffective against most Gram-negative bacteria. Moreover, teixobactin showed promising in vivo activity, resulting in a substantial reduction in bacterial burden in mice infected with methicillin-resistant $S$. aureus or Streptococcus pneumoniae. Further experiments revealed that the antibiotic inhibits peptidoglycan synthesis by targeting the peptidogycan precursor lipid II and the cell wall teichoic acid precursor lipid III. This explains why teixobactin is ineffective against Gram-negative bacteria, as they lack teichoic acids and have an outer membrane that shields peptidoglycan.

Importantly, the authors were unable to isolate teixobactin-resistant mutants, probably due to the fact that the drug has two targets, neither of which is a protein. The targeting of lipids rather than proteins is less likely to select for resistance as protein-encoding genes can evolve mutations that block the binding of antibiotics more readily than the emergence of lipid variants that preclude antibiotic binding. Whether resistance mechanisms already exist in nature is unclear, but as the producer of teixobactin is a Gramnegative species and is therefore intrinsically resistant, it is unlikely to encode a transferrable resistance mechanism for Gram-positive species.

This study is a proof-of-principle of the value of natural product discovery for identifying promising drug candidates, and the authors conclude that similar compounds with a low propensity to select for resistance await to be discovered.

Christina Tobin Kåhrström, Chief Editor, Nature Reviews Microbiology This article is modified from the original in Nature Rev. Microbiol. (http://dx.doi.org/10.1038/nrmicro3429)

ORIGINAL RESEARCH PAPER Ling, L. L. et al. A new antibiotic kills pathogens without detectable resistance. Nature http://dx.doi.org/ 10.1038/nature14098 (2015)

FURTHER READING Blair, J. M. A. et al. Molecular mechanisms of antibiotic resistance. Nature Rev. Microbiol. 13, 42-51 (2015) | Lewis, K. Platforms for antibiotic discovery. Nature Rev. Drug Discov. 12, 371-387 (2013) 